Ann. Biol. anim. Bioch. Biophys., 1977, 17 (4), 579-582.

\title{
Effets à long terme de la ligature du canal pancréatique sur la digestibilité apparente chez le porc
}

\author{
par T. CORRING, D. BOURDON
}

Laboratoire de Physiologie de la Nutrition, Station de Recherches sur l'Elevage des Porcs I.N.R.A., 78350 Jouy-en-Josas

Summary. Long-ferm effects of pancreatic duct ligature on digestibility in the pig.

The apparent digestibility of a diet was studied over a period of 87 days after ligature of the pancreatic duct in the growing pig. Faeces were collected during 2 periods of 10 days each. Throughout the experiment, mean daily gain of sham-operated pigs was higher $(560 \mathrm{~g} /$ day) than that of pancreatic duct-ligated animals $(421 \mathrm{~g} /$ day $)$. In the first period (15-24 days after ligature), the apparent digestibility of nitrogen was most affected (- 35.6 p. 100) when that of energy decreased by 12.1 p. 100 as compared to values cbtained in sham-operated subjects. Between periods 1 and 2 (78-87 days after ligature), apparent digestiblity of nitrogen increased by 20 p. 100 and that of energy by 6 p. 100 .

\section{Introduction.}

L'élimination de l'hydrolyse enzymatique pancréatique de la lumière intestinale entraîne une réduction de l'utilisation digestive. Cela a été montré dans le cas d'insuffisances pancréatiques (Säteri, 1975) ef par des études expérimentales, soit après ligature du ou des canaux pancréatiques (Douglas et al., 1953), soit après pancréatectomie totale (Shingleton et al., 1955), soit encore après dérivation de la sécrétion pancréatique (Corring et Bourdon, 1976).

Ainsi chez le porc (Anderson et Ash, 1971 ; Corring et Bourdon, 1976) la ligature du canal pancréatique ef la dérivation de la sécrétion pancréatique entraînent une diminution de la digestibilité apparente de l'azote de 17 p. 100 et de 13 p. 100 respectivement. Chez le lapin (Corring et Lebas, 1977), la digestibilité apparente de l'azote est réduite de 27,6 p. 100, 21 jours après ligature du canal pancréatique. Quelques études (Pekas, Hays et Thompson, 1964 ; Uram, Friedman et Kline, 1960) ont montré par ailleurs que les digestibilités étaient améliorées avec le temps, après suppression de l'hydrolyse enzymatique pancréatique.

Dans la présente expérience, nous avons étudié chez le porc en croissance, l'effet de la ligature du canal pancréatique sur l'utilisation digestive d'un aliment et son évolution a été suivie sur une durée prolongée. 


\section{Matériel et méthodes.}

Huit porcs mâles castrés, de race Large White, et pesant en moyenne $40 \mathrm{~kg}$ en début d'expérimentation, ont été utilisés. Après 8 jours d'adaptation au régime 4 porcs ont subi une ligature du canal de Wirsung (animaux $P$ ) et les 4 autres porcs ont subi une opération fictive (animaux TO).

La digestibilité apparente du régime, dont la composition est donnée dans le tableau 1, a été mesurée au cours de deux périodes de collecte de fèces de 10 jours chacune. La première période a débuté 15 jours après opération, la seconde période 78 jours après opération.

TABLEAU 1

Composition du régime expérimental

\begin{tabular}{|c|c|}
\hline & p. 100 matière sèche \\
\hline 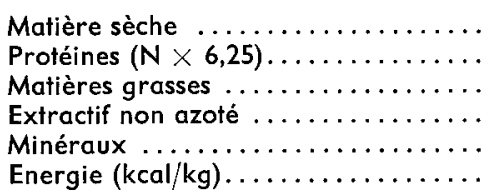 & $\begin{array}{r}88,04 \\
19,37 \\
2,11 \\
57,94 \\
6,28 \\
4444\end{array}$ \\
\hline
\end{tabular}

Les animaux ont ingéré la même quantité d'aliment (méthode du paired-feeding de Mitchell, 1930) qui a été de $1,51 \mathrm{~kg}$ de matière sèche au cours de la première période et de $1,98 \mathrm{~kg}$ de matière sèche pendant la seconde période.

Les critères utilisés pour estimer l'utilisation digestive ont été la digestibilité apparente de la matière sèche, de la matière organique, de l'énergie et de l'azote. Les animaux ont été pesés le premier et dernier jour de chacune des périodes de collecte de fèces.

\section{Résultats et discussion.}

Le gain de poids moyen quotidien des animaux T0 a été significativement supérieur ( $560 \mathrm{~g} /$ jour) à celui des porcs $P$ ( $421 \mathrm{~g} /$ jour) sur toute la durée de l'étude. Bien que réduite après ligature du canal pancréatique, la croissance des porcs est donc demeurée positive. Un résultat analogue a été trouvé chez le lapin (Corring et Lebas, 1977) mais non chez le ra! (Clowes et McPherson, 1951) et chez le poulet (Lepkovsky Nalbandov et Dimick, 1964) qui ont subi une perte de poids non compensée après suppression de l'hydrolyse enzymatique pancréatique.

Les digestibilités apparentes de la matière sèche, de la matière organique, de l'énergie et de l'azote sont rapportées dans le tableau 2. 
TABLEAU 2

Digestibilités apporentes après ligature du canal pancréalique chez le porc

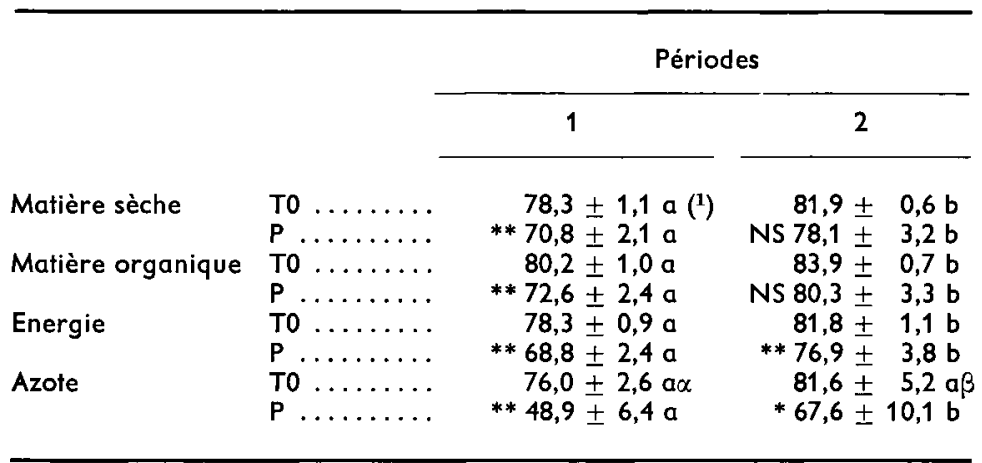

T0 : opération fictive; $P$ : canal pancréatique ligaturé.

(1) : écart-type de la moyenne.

Analyse statistique : Différence entre T0 et $P: * P<0,05 ; * * P<0,01$.

Différence entre périodes : les moyennes sur une même ligne suivies de lettres différentes sont significativement différentes.

$(\alpha, \beta: P<0,05 ; a, b: P<0,01)$.

Ces résultats montrent que l'utilisation digestive a été modifiée chez le porc en croissance, après ligature du canal pancréatique. Entre le $15^{\mathrm{e}}$ et $24^{\mathrm{e}}$ jour après opération, la digestibilité apparente de l'azote a été le plus affectée puisqu'elle a diminué de 35,6 p. 100 en moyenne. Ce résultat confirme ceux obtenus par ailleurs chez le porc. Selon Corring et Bourdon (1976) la digestibilité de l'azote a diminué de 13 p. 100 au cours des 5 jours qui ont suivi la dérivation de la sécrétion pancréatique et de 17 p. 100 selon Anderson et Ash (1971) entre la $10^{\mathrm{e}}$ et $22^{\mathrm{e}}$ semaine après ligature du canal de Wirsung. Chez d'autres espèces, telles que le rat, la diminution de la digestibilité apparente de l'azote a été de 22 p. 100 (Uram, Friedman et Kline, 1960) ou de 17 p. 100 (Clowes et McPherson, 1951).

En ce qui concerne les digestibilités apparentes de l'énergie, les résultats obtenus dans la présente expérience montrent qu'elles ont été également réduites mais dans des proportions moindres : - 12,1 p. 100 pour l'énergie et - 9,5 p. 100 pour la matière sèche et la matière organique. Ces résultats confirment également ceux obtenus chez le porc par Corring ef Bourdon (1976) et Anderson ef Ash (1971) et chez le lapin par Corring et Lebas (1977) et soulignent que l'utilisation digestive de l'énergie est peu affectée par la suppression de l'hydrolyse enzymatique pancréatique.

Lorsque l'on examine les valeurs des différentes digestibilités apparentes obtenues entre les $78^{\mathrm{e}}$ et $87 \mathrm{e}$ jours après opération, on constate qu'elles ont été améliorées par rapport à celles déterminées au cours de la première période de collecte de fèces. Ainsi, tenant compte de l'évolution des digestibilités apparentes chez les porcs T0, cette amélioration a été environ de $5 \mathrm{p}$. 100 pour la matière sèche ef la matière organique, de 6 p. 100 pour l'énergie et de 20 p. 100 pour l'azote.

Une amélioration de l'utilisation digestive après élimination de l'hydrolyse enzymatique pancréatique a été observée sur une plus courte période chez le porcelet 
(Pekas, Hays et Thompson, 1964), le rat (Uram, Friedman et Kline, 1960) et le lapin (Corring et Lebas, 1977). Ainsi, chez le porcelet, la digestibilité apparente de l'azote a été améliorée de 32 p. 100 en moyenne entre la $2^{\mathrm{e}}$ et $8 \mathrm{e}$ semaine après ligature du canal pancréatique effectuée à l'âge de 2 jours. Chez le rat, la digestibilifé apparente de l'azote d'origine végétale a augmenté de $32 \mathrm{p} .100$ entre le $3^{\mathrm{e}}$ et $40^{\mathrm{e}}$ jour après opération. Chez le lapin, elle a augmenté de 6,3 p. 100 entre le 16 et $46 \mathrm{e}$ jour après opération.

Cette amélioration traduit la mise en place d'une compensation digestive dont l'origine reste inconnue. En ce qui concerne l'utilisation digestive de l'énergie, l'étude de Senegas et al. (1976) laisse supposer qu'une stimulation de l'amylase intestinale après ligature du canal pancréatique serait à l'origine de la compensation digestive. Selon Catala et Bonnafous (1974) cette compensation peut être également d'origine bactérienne.

Commission CNERNA Digestion-Absorption, Tours, 13 novembre 1976.

\section{Références}

ANDERSON D. M., ASH R. W., 1971. The effect of ligating the pancreatic duct on digestion in the pig. Proc. Nutr. Soc., 30, $34 \mathrm{~A}$.

CATALA J., BONNAFOUS R., 1974. Variations quantitatives de la microflore intestinale du lapin après ligature du canal pancréatique. C. R. Acad. Sci. Paris, Ser. D, 278, 3351-3353.

CLOWES G. H. A. Jr., McPHERSON L. B., 1951. Production of fatty livers by ligature of the pancreatic duct in Rats. Am. J. Physiol., 165, 628-638.

CORRING T., BOURDON D., 1976. Short-dated influence of removal of the exocrine pancreatic secretion (enzymatic or total) upon the apparent digestibility of a diet in the pig. Nutr. Rep. int. 14, 621-627.

CORRING T., LEBAS F., 1977. Effets à court et moyen terme de la ligature du canal pancréatique sur la digestibilité d'un aliment, chez le lapin en croissance et soumis à une alimentation restreinte. Ann. Biol. anim. Bioch. Biophys. 17, 299-307.

DOUGLAS G. J., REINAUER A. J., BROOKS W. C., PRATT J. H., 1953. The effect on digestion and absorption of excluding the pancreatic juice from the intestine. Gastroenterology, 23, 452-459.

LEPKOVSKY S., NALBANDOV A. V., DIMICK M. K., 1964. Growth and reproduction of depancreatized chickens. Endocrinology, 74, 207-221.

MITCHELL H. H., 1930. The paired-feeding method : its value and limitation in livestock experimentation. Proc. amer. Soc. Anim. Prod., 63-73.

PEKAS J. C., HAYS V. W., THOMPSON A. M., 1964. Exclusion of the exocrine pancreatic secretion. Effect on digestibility of soybean and milk protein by baby pigs at various ages. J. Nutr., 82, 277-286.

SÄTERI H., 1975. Investigations of the exocrine pancreatic function in dogs suffering from chronic exocrine pancreatic insuff iciency. Acta vet. scand, supp. 53, 1-86.

SENEGAS F., BALAS D., FREXINOS J., RIBET A., 1976. Effects of pancreatic duct ligation on the secretory processes of the hamster intestinal mucosa. Scand. J. Gastroenterol. Abstracts of papers presented at the Ninth Symposium of the European Pancreatic Club, OSLO, Norway, 14-16 october 1976, 11, suppl. 41, 47.

SHINGLETON W. W., WELLS M. H., BAYLIN G. H., RUFFIN J. M., SAUNDERS A., DURHAM N. C., 1955. The use of radioactive-labelled protein and fat in the evaluation of pancreatic disorders. Surgery, 38, 134-142.

URAM J. A., FRIEDMAN L., KLINE O. L., 1960. Relation of pancreatic exocrine to nutrition of the rat. Am. J. Physiol., 199, 387-394. 